\title{
Prognosis of metastatic breast cancer: are there differences between patients with de novo and recurrent metastatic breast cancer?
}

D J A Lobbezoo ${ }^{*, 1,2}$, R J W van Kampen ${ }^{1}$, A C Voogd ${ }^{1,3}$, M W Dercksen ${ }^{2}$, F van den Berkmortel ${ }^{4}$, T J Smilde ${ }^{5}$, A J van de Wouw ${ }^{6}$, F P J Peters ${ }^{7}$, J M G H van Riel ${ }^{8}$, N A J B Peters ${ }^{9}$, M de Boer ${ }^{1}$, P G M Peer ${ }^{10}$ and V C G Tjan-Heijnen ${ }^{1}$

${ }^{1}$ GROW- School for Oncology and Developmental Biology, Maastricht University Medical Centre, Maastricht, The Netherlands; ${ }^{2}$ Department of Internal Medicine, Máxima Medical Centre, Veldhoven, The Netherlands; ${ }^{3}$ Department of Research, Comprehensive Cancer Centre, Eindhoven, The Netherlands; ${ }^{4}$ Department of Internal Medicine, Atrium Medical Centre Parkstad, Heerlen, The Netherlands; ${ }^{5}$ Department of Internal Medicine, Jeroen Bosch Hospital, Den Bosch, The Netherlands; ${ }^{6}$ Department of Internal Medicine, VieCuri Medical Centre, Venlo, The Netherlands; ${ }^{7}$ Department of Internal Medicine, Orbis Medical Centre, Sittard, The Netherlands; ${ }^{8}$ Department of Internal Medicine, St Elisabeth Hospital, Tilburg, The Netherlands; ${ }^{9}$ Department of Internal Medicine, St Jans Hospital, Weert, The Netherlands and ${ }^{10}$ Department for Health Evidence, Radboud university medical centre, Nijmegen, The Netherlands

Background: We aimed to determine the prognostic impact of time between primary breast cancer and diagnosis of distant metastasis (metastatic-free interval, MFI) on the survival of metastatic breast cancer patients.

Methods: Consecutive patients diagnosed with metastatic breast cancer in 2007-2009 in eight hospitals in the Southeast of the Netherlands were included and categorised based on MFI. Survival curves were estimated using the Kaplan-Meier method. Cox proportional hazards model was used to determine the prognostic impact of de novo metastatic breast cancer vs recurrent metastatic breast cancer (MFI $\leqslant 24$ months and > 24 months), adjusted for age, hormone receptor and HER2 status, initial site of metastasis and use of prior (neo)adjuvant systemic therapy.

Results: Eight hundred and fifteen patients were included and divided in three subgroups based on MFI; 154 patients with de novo metastatic breast cancer, 176 patients with MFI $<24$ months and 485 patients with MFI $>24$ months. Patients with de novo metastatic breast cancer had a prolonged survival compared with patients with recurrent metastatic breast cancer with MFI $<24$ months (median 29.4 vs 9.1 months, $P<0.0001$ ), but no difference in survival compared with patients with recurrent metastatic breast cancer with MFI $>24$ months (median, 29.4 vs 27.9 months, $P=0.73$ ). Adjusting for other prognostic factors, patients with MFI $<24$ months had increased mortality risk (hazard ratio 1.97, 95\% Cl 1.49-2.60, $P<0.0001$ ) compared with patients with de novo metastatic breast cancer. When comparing recurrent metastatic breast cancer with MFI > 24 months with de novo metastatic breast cancer no significant difference in mortality risk was found. The association between MFI and survival was seen irrespective of use of (neo)adjuvant systemic therapy.

Conclusion: Patients with de novo metastatic breast cancer had a significantly better outcome when compared with patients with MFI $<24$ months, irrespective of the use of prior adjuvant systemic therapy in the latter group. However, compared with patients with MFI > 24 months, patients with de novo metastatic breast cancer had similar outcome.

*Correspondence: DJA Lobbezoo; E-mail: d.lobbezoo@mmc.nl

Received 9 January 2015; revised 1 March 2015; accepted 9 March 2015; published online 16 April 2015

(c) 2015 Cancer Research UK. All rights reserved 0007-0920/15 
Despite progress in the treatment of early breast cancer, $20-30 \%$ of the patients will develop a distant recurrence (Harris et al, 1993). Once distant recurrence has occurred the disease remains largely incurable and median survival of patients with metastatic breast cancer ranges from 2 to 3 years (Cardoso et al, 2012).

The outcome of patients with metastatic breast cancer depends on several prognostic factors. Biological breast cancer subtypes based on hormone receptor (HR) and human epidermal growth factor receptor 2 (HER2) status are associated with different outcomes once distant recurrence has occurred (Kennecke et al, 2010; Lobbezoo et al, 2013). Besides biological breast cancer subtype, other known prognostic factors are age, performance status and site and number of distant metastases (Yamamoto et al, 1998; Largillier et al, 2008).

Furthermore, the metastatic-free interval (MFI), which is defined as the time between primary breast cancer diagnosis and the development of distant recurrence, is a known strong prognostic factor for survival of patients with metastatic breast cancer. Metastatic-free interval $<24$ months is associated with a worse prognosis, as shown in several population-based studies of patients with metastatic breast cancer (Largillier et al, 2008; Puente et al, 2010; Llombart-Cussac et al, 2014). The importance of MFI is stressed by the incorporation of this prognostic factor in clinically relevant and validated prognostic models for patients with metastatic breast cancer (Yamamoto et al, 1998; Regierer et al, 2014).

Acknowledging separate prognostic groups based on MFI, patients with distant metastasis at initial breast cancer diagnosis can also be viewed as a distinct prognostic subgroup. This subgroup, called de novo metastatic breast cancer, could on the one hand be suggested to represent a poor prognostic group with early distant metastasis as a sign of more aggressive disease. On the other hand, one could hypothesise that patients with de novo metastatic breast cancer have a better prognosis because their disease is therapy-naive and thus less likely to show resistance to systemic therapy.

Indeed, in a large study on metastatic breast cancer diagnosed in the period 1992-2007, outcome of patients with de novo metastatic breast cancer was superior compared with patients with recurrent metastatic breast cancer (Dawood et al, 2010b). This favourable outcome was also demonstrated for patients with de novo HER2 positive metastatic breast cancer, as compared to those with recurrent HER2 positive metastatic breast cancer (Yardley et al, 2014). However, in another analysis on HER2-positive patients all treated with first-line palliative trastuzumab-based therapy no difference in outcome between de novo and recurrent metastatic breast cancer was found (Rossi et al, 2014).

This led to our study investigating the prognosis and prognostic factors, including HR and HER2 status, for patients with de novo metastatic breast cancer compared with patients with recurrent metastatic breast cancer in a large multicentre study. All patients were diagnosed with metastatic breast cancer in the period 20072009 and were treated according to modern treatment strategies, including targeted therapy.

\section{PATIENTS AND METHODS}

Patients and data collection. All consecutive patients diagnosed with metastatic breast cancer in 2007-2009 in eight hospitals in the Southeast of the Netherlands were identified. Patients were included irrespective of the date of diagnosis of the primary tumour with the exception of patients with a diagnosis of primary breast cancer before 1990, due to the limited available data on the primary tumour characteristics and the initial treatment.

Data on all included patients was obtained from medical files by specially trained registration clerks, after approval from the Medical Research Ethics Committee of Maastricht University
Medical Centre. Information was collected on patient and tumour characteristics, treatment information (surgery, radiotherapy and systemic treatment, both adjuvant and palliative), number and sites of distant metastases and survival time. Initial metastatic sites were registered as single or multiple and were categorised as: bone, visceral (including lung, liver, pleural, peritoneal, pericardial and lymphangitic carcinomatosis), brain (including leptomeningeal and central nervous system), skin and lymph nodes and multiple metastases (more than one of the aforementioned metastatic sites). Tumours were characterised by the sixth edition of the TNM classification of malignant tumours (Greene et al, 2002) and Scarff Bloom Richardson (SBR) histological grading (Elston and Ellis, 1991). We used pathological TNM also for the small amount of patients with neoadjuvant systemic therapy ( $9 \%$ of all patients treated with (neo)adjuvant therapy). Oestrogen receptor and progesterone receptor positivity was defined as positive nuclear staining of $\geqslant 10 \%$ and HR positivity was defined as Oestrogen receptor- and/or progesterone receptor-positive breast cancer. HER2 positivity was defined as immunohistochemistry score of $3+$, or $2+$ with a positive fluorescence in situ hybridisation result. In case of missing HER2 status a dedicated pathologist centrally reviewed missing data when material was available.

Data analysis. We categorised the total of 815 included metastatic breast cancer patients based on MFI. Metastatic-free interval was defined as time between date of diagnosis of primary breast cancer and date of diagnosis of first distant metastatic recurrence. On the basis of the cutoff points used in other studies and their demonstrated differential impact, patients were divided in three categories; patients with de novo metastatic breast cancer (MFI $<3$ months), patients with recurrent metastatic breast cancer with MFI $\leqslant 24$ months and patients with recurrent metastatic breast cancer with MFI of $>24$ months.

The primary aim of our study was to assess differences in survival of patients with de novo metastatic breast cancer compared with those with recurrent metastatic breast cancer. Survival of patients with distant metastasis was defined as time between date of diagnosis of first distant metastasis and date of death. All patients still alive were censored at the date of last follow-up of each individual patient. Survival curves were obtained using the Kaplan-Meier method.

We selected prognostic factors based on clinical importance rather than by statistical significance and included the following factors in the model; age at metastatic breast cancer diagnosis, HR and HER2 status and initial number and site of metastases. First, a Cox proportional hazards model was used to assess whether the prognostic impact of these factors differed between the three subgroups based on MFI by statistical testing of the interaction between the prognostic factors and MFI with a likelihood ratio test. Second, the ratios of the hazard rates comparing de novo metastatic breast cancer with recurrent metastatic breast cancer were adjusted for these factors in a Cox regression model. To evaluate the effect of (neo)adjuvant therapy we repeated this model when excluding patients with (neo)adjuvant systemic therapy.

All reported P-values were two-sided and a $P$-value $<0.05$ was considered of statistical significance.

\section{RESULTS}

Characteristics. A total of 815 metastatic breast cancer patients were included in the study, of which 154 patients (19\%) were diagnosed with de novo metastatic breast cancer and 661 patients (81\%) had recurrent metastatic breast cancer (Table 1). Of the patients with recurrent metastatic breast cancer, 176 patients $(27 \%)$ had MFI $<24$ months and 485 patients $(73 \%)$ had MFI $>24$ months. 
Median age at diagnosis of metastatic breast cancer was 61 years (range 25-89 years) for patients with de novo metastatic breast cancer and 64 years (range 25-93 year) for patients with recurrent metastatic breast cancer, irrespective of MFI $(P=0.06)$.

Pathological tumour and lymph node status was missing for the majority of patients with de novo metastatic breast cancer, as surgical removal of the primary tumour was not routinely performed after diagnosis of metastatic breast cancer $(83 \%$ of patients with de novo metastatic breast cancer did not have surgery of the primary tumour).

Hormone receptor-positive breast cancer was most frequently seen in patients with de novo metastatic breast cancer $(81 \%)$ and recurrent metastatic breast cancer with MFI > 24 months (83\%), but less in patients with recurrent metastatic breast cancer with MFI $<24$ months $(55 \%)(P<.0001)$. Human epidermal growth factor receptor 2-positive status was not different between patients with de novo metastatic breast cancer (23\%), recurrent metastatic breast cancer with MFI $>24$ months $(18 \%)(P=0.21)$ and recurrent metastatic breast cancer with MFI $<24$ months $(21 \% ; P=0.76)$.

The percentage of patients with visceral or brain metastasis as initial metastatic site for recurrent metastatic breast cancer with MFI $<24$ months was, respectively, 33\% and 10\%. These percentages were lower for patients with de novo metastatic breast cancer (respectively, 24\% and 0\%) and for patients with recurrent metastatic breast cancer with MFI > 24 months (respectively, 27\% and 2\%).

For all patients with recurrent metastatic breast cancer, $46 \%$ had received prior (neo) adjuvant chemotherapy and 51\% prior (neo-) adjuvant endocrine therapy.

Of patients with de novo metastatic breast cancer 57\% received at least one-line of palliative chemotherapy vs 53\% of patients with recurrent metastatic breast cancer $(P=0.35)$. Also the use of any palliative endocrine therapy was not different between de novo and recurrent metastatic breast cancer (respectively, 69\% vs 62\%; $P=0.10)$.

Survival and prognostic factors. Median follow-up after diagnosis of metastatic disease was 37.1 months (range 2.1-55.4), with 302 patients (37\%) being alive at the end of the follow-up period.

The median survival of patients with de novo metastatic breast cancer was 29.4 months (95\% CI 19.3-35.0 months) compared with 21.1 months (95\% CI 18.7-24.4 months) for patients with recurrent metastatic breast cancer $(P=0.14)$.

When looking at the patients with recurrent metastatic breast cancer stratified by MFI, survival of those with de novo metastatic breast cancer was significantly better than for patients with MFI <24 months (median, 29.4 vs 9.1 months, $P<.0001$ ) (Figure 1). Survival of de novo metastatic breast cancer patients was not significantly different from the survival of recurrent metastatic breast cancer patients with MFI of $>24$ months (median, 29.4 vs 27.9 months, $P=0.73$ ) (Figure 1).

The prognostic impact of the prognostic factors did not differ significantly for the three MFI groups (test for interaction $P=0.98)$. Therefore, the results could be pooled to obtain the final Cox proportional hazards model (Table 2).

Adjusted for other prognostic factors, patients with MFI $<24$ months had a significantly increased risk in mortality (hazard ratio 1.97; 95\% CI 1.49-2.60) compared with patients with de novo metastatic cancer (Table 2). Recurrent metastatic breast cancer patients with MFI > 24 months had no increased risk in mortality compared with de novo metastatic breast cancer patients (hazard ratio of 0.89 , 95\% CI 0.70-1.14) (Table 2).

Older age at diagnosis of metastatic breast cancer, HR-negative breast cancer, HER2-negative breast cancer, multiple initial metastatic sites as well as brain or visceral metastases as initial site compared with bone metastases were all associated with a worse prognostic impact on outcome (Table 2).
Performing the multivariable analysis when excluding patients with (neo)adjuvant systemic therapy, thereby comparing the therapy-naive patients with recurrent metastatic breast cancer and de novo metastatic breast cancer, the increased mortality risk for patients with MFI $<24$ months compared with de novo metastatic breast cancer remained statistically significant (adjusted hazard ratio $1.69,95 \%$ CI 1.11-2.58) (Table 2).

\section{DISCUSSION}

We present survival and prognostic factors of patients with de novo metastatic breast cancer compared with patients with recurrent metastatic breast cancer in a multi-centre, unselected cohort of patients. As all patients were diagnosed with metastatic breast cancer between 2007 and 2009, this cohort reflects a time period in which HER2 status was routinely performed and treatment included targeted therapy.

There was no significant difference in prognosis between patients with de novo and recurrent metastatic breast cancer irrespective of MFI.

The median overall survival of 29 months for patients with de novo metastatic breast cancer in this cohort was comparable to the survival found in other cohort studies on patients with de novo metastatic breast cancer (Andre et al, 2004; Dawood et al, 2010b; Pal et al, 2012).

However, when stratifying patients with recurrent metastatic breast cancer according to the MFI differences in survival between de novo and recurrent metastatic breast cancer became apparent. Patients with de novo metastatic breast cancer had a similar outcome when compared with patients with late distant recurrent metastatic breast cancer. However, patients with de novo metastatic breast cancer had a significant better outcome when compared with patients with an early distant recurrence, also after exclusion of the patients receiving (neo)adjuvant systemic treatment.

We defined early distant recurrence as MFI shorter than 24 months, based on the cutoff points used in other studies and the demonstrated prognostic impact (Yamamoto et al, 1998; Largillier et al, 2008; Puente et al, 2010; Llombart-Cussac et al, 2014; Regierer et al, 2014).

When looking closer at this subgroup, patients with an early distant recurrence were more likely to have HR-negative breast cancer. It is known that breast cancer subtypes, based on HR and HER2 status, have different prognostic impact and different patterns of distant recurrence, with HR-negative subgroups having earlier recurrence and unfavourable prognosis compared with HRpositive subgroups (Kennecke et al, 2010; Lobbezoo et al, 2013). However, even when adjusting for HR-negative status, the difference in survival between de novo and early distant recurrent metastatic breast cancer remained present.

Another hypothesis explaining the better outcome of de novo metastatic breast cancer compared with early recurrent metastatic breast cancer is the use of adjuvant systemic therapy in patients with recurrent metastatic breast cancer. This could lead to more resistant metastatic disease compared with patients with therapynaive de novo metastatic breast cancer. But, when we excluded patients with recurrent metastatic breast cancer with (neo)adjuvant systemic therapy, the difference in survival between de novo and early distant recurrent metastatic breast cancer remained present. Hence, in our cohort there was no evidence that the administration of systemic therapy in early breast cancer could explain the better outcome of de novo metastatic breast cancer when compared with patients with an early distant recurrence.

The role of surgery of the primary breast tumour on the outcome of metastatic breast cancer patients remains controversial. Initial retrospective studies and meta-analyses of these data 
Table 1. Baseline characteristics

\begin{tabular}{|c|c|c|c|c|c|c|c|c|c|c|c|}
\hline \multirow{3}{*}{ Characteristics } & \multicolumn{2}{|c|}{ De novo } & \multicolumn{2}{|c|}{ Recurrent all } & \multirow{2}{*}{\multicolumn{3}{|c|}{\begin{tabular}{|c|}
$\begin{array}{c}\text { Recurrent with } \\
\text { MFI } \leqslant 24 \text { months }\end{array}$ \\
$n=176$
\end{tabular}}} & \multirow{2}{*}{\multicolumn{3}{|c|}{\begin{tabular}{|c}
$\begin{array}{c}\text { Recurrent with } \\
\text { MFI }>24 \text { months }\end{array}$ \\
$n=485$
\end{tabular}}} & \multirow{3}{*}{$P$-value } \\
\hline & \multicolumn{2}{|c|}{$n=154$} & \multicolumn{2}{|c|}{$n=661$} & & & & & & & \\
\hline & No. & $\%$ & No. & $\%$ & $P$-value & No. & $\%$ & $P$-value & No. & $\%$ & \\
\hline \multicolumn{12}{|c|}{ Age at diagnosis of metastatic disease } \\
\hline Median, (range) & 61 & $25-89$ & 64 & $25-93$ & \multirow[t]{4}{*}{0.06} & 62 & $25-93$ & \multirow[t]{4}{*}{0.40} & 64 & $32-90$ & \multirow[t]{4}{*}{0.04} \\
\hline$<40$ years & 10 & 6 & 18 & 3 & & 12 & 7 & & 6 & 1 & \\
\hline $40-70$ years & 100 & 65 & 408 & 62 & & 98 & 56 & & 310 & 64 & \\
\hline$>70$ years & 44 & 29 & 235 & 35 & & 66 & 37 & & 169 & 35 & \\
\hline Primary tumour & $g e^{a}$ & & & & & & & & & & \\
\hline T1 & 9 & 25 & 240 & 44 & & 38 & 31 & & 202 & 48 & \\
\hline T2 & 22 & 63 & 265 & 48 & & 73 & 58 & & 192 & 45 & \\
\hline T3 & 2 & 6 & 29 & 5 & & 8 & 6 & & 21 & 5 & \\
\hline $\mathrm{T} 4$ & 2 & 6 & 16 & 3 & & 6 & 5 & & 10 & 2 & \\
\hline Unknown & 119 & & 111 & & & 51 & & & 60 & & \\
\hline Regional lymph & le stac & rimary & mour ${ }^{a}$ & & & & & & & & \\
\hline No & 6 & 17 & 230 & 41 & & 46 & 37 & & 184 & 43 & \\
\hline N1 & 13 & 37 & 187 & 34 & & 32 & 25 & & 155 & 36 & \\
\hline N2 & 6 & 17 & 82 & 15 & & 26 & 21 & & 56 & 13 & \\
\hline N3 & 10 & 29 & 57 & 10 & & 22 & 17 & & 35 & 8 & \\
\hline Unknown & 119 & & 105 & & & 50 & & & 55 & & \\
\hline Histological gra & f prin & tumou & & & & & & & & & \\
\hline SBR 1 & 8 & 11 & 55 & 11 & 0.26 & 10 & 6 & 0.01 & 45 & 14 & 0.59 \\
\hline SBR 2 & 39 & 53 & 212 & 44 & & 57 & 37 & & 155 & 47 & \\
\hline SBR 3 & 26 & 36 & 219 & 45 & & 89 & 57 & & 130 & 39 & \\
\hline Unknown & 81 & & 175 & & & 20 & & & 155 & & \\
\hline Hormone recep & status & & & & & & & & & & \\
\hline Positive & 121 & 81 & 490 & 76 & 0.21 & 96 & 55 & $<0.0001$ & 394 & 83 & 0.43 \\
\hline Negative & 29 & 19 & 156 & 24 & & 78 & 45 & & 78 & 17 & \\
\hline Unknown & 4 & & 15 & & & 2 & & & 13 & & \\
\hline HER2 status & & & & & & & & & & & \\
\hline Positive & 34 & 23 & 122 & 19 & 0.29 & 37 & 21 & 0.76 & 85 & 18 & 0.21 \\
\hline Negative & 116 & 77 & 524 & 81 & & 137 & 79 & & 387 & 82 & \\
\hline Unknown & 4 & & 15 & & & 2 & & & 13 & & \\
\hline Prior (neo)adju & chem & rapy & & & & & & & & & \\
\hline Yes & - & & 306 & 46 & & 85 & 48 & & 221 & 46 & \\
\hline No & & & 355 & 54 & & 91 & 52 & & 264 & 54 & \\
\hline Prior (neo) adju & endo & e ther & & & & & & & & & \\
\hline Yes & - & & 339 & 51 & & 64 & 36 & & 275 & 57 & \\
\hline No & & & 322 & 49 & & 112 & 64 & & 210 & 43 & \\
\hline Prior (neo) adju & anti- & 2 thera & & & & & & & & & \\
\hline Yes & - & & 44 & 7 & & 17 & 10 & & 27 & 6 & \\
\hline No & & & 617 & 93 & & 159 & 90 & & 458 & 94 & \\
\hline No. of metasta & tes & & & & & & & & & & \\
\hline 1 & 103 & 67 & 426 & 64 & 0.57 & 123 & 70 & 0.56 & 303 & 62 & 0.32 \\
\hline$\geqslant 2$ & 51 & 33 & 235 & 36 & & 53 & 30 & & 182 & 38 & \\
\hline Site of metasta & & & & & & & & & & & \\
\hline Bone & 60 & 39 & 202 & 31 & 0.04 & 41 & 23 & 0.002 & 161 & 33 & 0.19 \\
\hline Visceral & 37 & 24 & 188 & 28 & 0.27 & 58 & 33 & 0.07 & 130 & 27 & 0.49 \\
\hline Brain & 0 & 0 & 26 & 4 & 0.01 & 18 & 10 & $<0.0001$ & 8 & 2 & 0.11 \\
\hline Skin and lymph & 9 & 6 & 46 & 7 & 0.62 & 16 & 9 & 0.27 & 30 & 6 & 0.88 \\
\hline nodes & & & & & & & & & & & \\
\hline Multiple & 48 & 31 & 199 & 30 & 0.80 & 43 & 25 & 0.17 & 156 & 32 & 0.82 \\
\hline Any palliative $c$ & other & & & & & & & & & & \\
\hline Yes & 88 & 57 & 350 & 53 & 0.35 & 84 & 48 & 0.09 & 266 & 55 & 0.62 \\
\hline No & 66 & 43 & 311 & 47 & & 92 & 52 & & 219 & 45 & \\
\hline Any palliative $\mathrm{e}$ & crine $t$ & apy & & & & & & & & & \\
\hline Yes & 106 & 69 & 408 & 62 & 0.10 & 67 & 38 & $<0.0001$ & 341 & 70 & 0.73 \\
\hline No & 48 & 31 & 253 & 38 & & 109 & 62 & & 144 & 30 & \\
\hline Any palliative $t$ & ted th & & & & & & & & & & \\
\hline Yes & 33 & 21 & 121 & 18 & 0.37 & 23 & 13 & 0.04 & 98 & 20 & 0.74 \\
\hline No & 121 & 79 & 540 & 82 & & 153 & 87 & & 387 & 80 & \\
\hline
\end{tabular}


supported the idea that surgery of the primary tumour in de novo metastatic breast cancer could have a positive impact on outcome (Ruiterkamp et al, 2010; Ali and Le Scodan, 2011; Harris et al, 2013). However, data on randomised clinical trials could not show a survival advantage for surgery of the primary tumour and palliative systemic therapy compared with palliative systemic therapy alone (Badwe et al, 2013; Soran et al, 2013). Furthermore, when looking at the current views on molecular models of metastasis and tumour dormancy, some hypothesise that surgery of the primary tumour can be the systemic event that interrupts the dormant state of metastatic cancer cells, and thereby even have an unfavourable effect on outcome (Dillekas et al, 2014).

The model of metastasis in breast cancer has evolved over the years and the knowledge gathered by genomic assays supports the idea that the capacity of metastasis is acquired early in the life of primary breast cancer (Weigelt et al, 2005; Chiang and Massague, 2008). Therefore, the assessment of recurrence risk is not only dependent on classical clinicopathological factors, but also on detection of metastatic potential of the primary breast tumour. This also leads to newer insights into relevant prognostic factors in early breast cancer to determine risk of recurrence, amongst others gene-expression profiling and the detection of disseminated

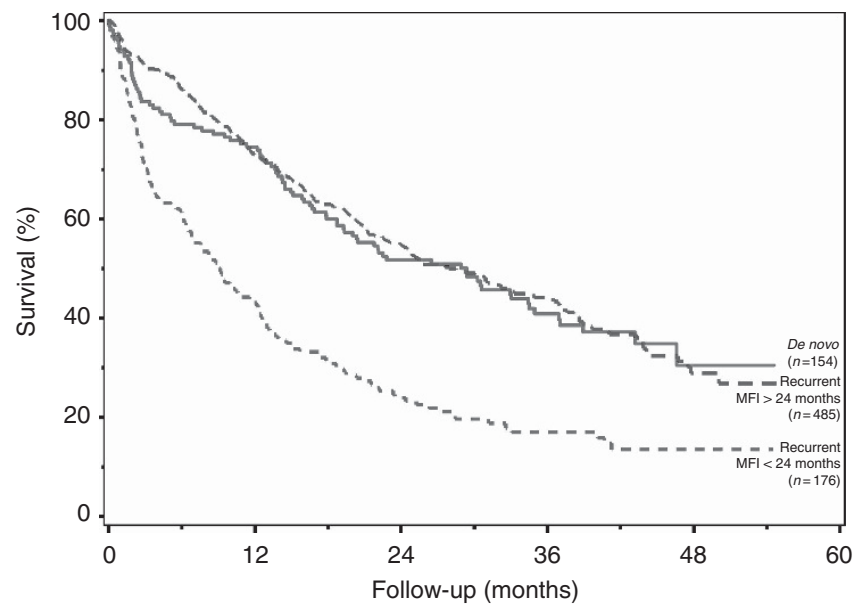

Figure 1. Survival after diagnosis of metastatic breast cancer for patients with de novo metastatic breast cancer and recurrent metastatic breast cancer with MFI $<24$ months and recurrent metastatic breast cancer with MFI $>24$ months. tumour cells. However, once metastatic breast cancer is present, the need for clinical prognostic factors remains and is of importance to guide further treatment decision and to provide prognostic information to patients.

In this analyses, prognostic factors that influenced survival besides MFI were age at diagnosis of metastatic breast cancer, HR and HER2 status and initial site of metastases. This is in accordance with other studies investigating prognostic factors in metastatic breast cancer (Andre et al, 2004; Largillier et al, 2008; Dawood et al, 2010b; Lobbezoo et al, 2013). As expected, we found a favourable impact of HR-positive breast cancer, with a hazard ratio of 0.57 $(P<0.0001)$ compared with HR-negative breast cancer in our cohort, which is similar to that found in a large study on survival between de novo and recurrent metastatic breast cancer (Dawood et al, 2010b). Hormone receptor-positivity is a known favourable prognostic factor as well as a predictive factor for response to endocrine therapy. The hazard ratio as calculated in our model is not only reflecting the favourable prognostic influence but also the strong predictive influence for response to endocrine therapy.

The strength of this study is the incorporation of HER2 status, due to a very low rate of missing results for HER2 status (only 2\%) because all missing HER2 results were centrally determined whenever possible. Interestingly, HER2 positive breast cancer was found to have a favourable influence on outcome of metastatic breast cancer. Even though in the pretrastuzumab era, the amplification of HER2 in breast cancer was demonstrated to be a strong unfavourable prognostic factor (Slamon et al, 1987). However, with the availability of anti-HER2 therapy this unfavourable prognostic factor has also become a favourable predictive factor for response to anti-HER2 therapy. And with the implementation of anti-HER2 therapy, outcome of HER2-positive breast cancer has changed to the extent that HER2-positive status is nowadays a prognostic factor associated with a favourable outcome in breast cancer (Dawood et al, 2010a; Lobbezoo et al, 2013). Numbers were too low to look at outcome of biological subtypes based on HR and HER2 in the three separate subgroups, but previous studies investigated differences in outcome between HER2-positive de novo metastatic breast cancer and HER2-positive recurrent metastatic breast cancer. In an unplanned analysis from the registHER observational study, a better survival was found for patients with HER2 positive de novo metastatic breast cancer compared with patients with HER2-positive recurrent metastatic breast cancer (Yardley et al, 2014). This difference in survival was seen regardless of adjuvant systemic therapy for patients with recurrent breast cancer. However, only $9 \%$ of patients with HER2positive recurrent breast cancer had received anti-HER2 therapy in

Table 2. Multivariable analysis for survival after diagnosis of metastatic breast cancer

\begin{tabular}{|c|c|c|c|c|c|c|}
\hline & \multicolumn{3}{|c|}{ All patients } & \multicolumn{3}{|c|}{ Patients without (neo)adjuvant systemic therapy } \\
\hline & \multicolumn{3}{|c|}{$n=815$} & \multicolumn{3}{|c|}{$n=318$} \\
\hline & Hazard ratio & $95 \% \mathrm{Cl}$ & $P$-value & Hazard Ratio & $95 \% \mathrm{Cl}$ & $P$-value \\
\hline Recurrent with MFI $<24$ months vs de novo & 1.97 & $1.49-2.60$ & $<0.0001$ & 1.69 & $1.11-2.58$ & 0.015 \\
\hline Recurrent with MFI $>24$ months vs de novo & 0.89 & $0.70-1.14$ & 0.358 & 0.89 & $0.64-1.24$ & 0.498 \\
\hline Age at diagnosis of metastatic breast cancer & 1.02 & $1.01-1.02$ & $<0.0001$ & 1.02 & $1.00-1.03$ & 0.008 \\
\hline Hormone receptor negative vs positive & 1.74 & $1.40-2.17$ & $<0.0001$ & 2.21 & $1.58-3.11$ & $<0.0001$ \\
\hline HER2 negative vs positive & 1.44 & $1.13-1.83$ & 0.003 & 1.27 & $0.88-1.86$ & 0.207 \\
\hline Visceral metastases as initial metastatic site vs bone & 1.80 & $1.41-2.31$ & $<0.0001$ & 1.56 & $1.03-2.36$ & 0.037 \\
\hline Brain metastases as initial metastatic site vs bone & 2.31 & $1.40-3.80$ & 0.001 & 4.50 & $1.49-13.64$ & 0.008 \\
\hline Skin/lymph nodes as initial metastatic site vs bone & 1.06 & $0.71-1.59$ & 0.783 & 1.37 & $0.71-2.67$ & 0.350 \\
\hline Multiple initial metastatic sites vs 1 metastatic site & 2.30 & $1.81-2.93$ & $<0.0001$ & 2.66 & $1.83-3.87$ & $<0.0001$ \\
\hline
\end{tabular}


adjuvant setting. Another study investigating the outcome of patients with HER2-positive de novo metastatic breast cancer did not demonstrate a difference in outcome compared with patients with HER2-positive recurrent metastatic breast cancer (Rossi et al, 2014).

Besides low number of patients when dividing the total cohort according to biological breast cancer subtype, there are other limitations of this study owing to its retrospective nature. Leadtime bias due to improved imaging could be a confounder in this study. However, all patients were diagnosed with metastatic breast cancer (either de novo or recurrent) during the same recent and relatively short time period so the availability of imaging techniques was probably equal. Even so, with the inclusion of all patients with metastatic breast cancer diagnosed in eight different hospitals we feel this cohort is a good representation of the actual diagnosis and treatment of patients with metastatic breast cancer in the Netherlands. With the availability and implementation of more advanced and sensitive diagnostic techniques, for example, positron emission tomography, the subgroup of patients with de novo metastatic breast cancer will probably increase. Therefore, prognostic information on this subgroup is of relevance for oncology practice.

In conclusion, we confirm that MFI is a strong prognostic indicator for outcome of metastatic breast cancer patients, with a short MFI being prognostic unfavourable. However, de novo metastatic breast cancer, which can be considered as a group with a 'very short' MFI was not found to be a very poor prognostic subgroup. In real life, patients with de novo metastatic breast cancer had a clearly better outcome, similar to what was seen in patients with metastatic breast cancer with MFI > 24 months, compared with patients with MFI $<24$ months who had a nearly two-fold increased mortality risk.

\section{ACKNOWLEDGEMENTS}

We thank Wim A.J.G. Lemmens for his assistance with performing the statistical analyses. This study was funded by the Netherlands Organization for Health Research and Development (ZonMw: 80-82500-98-8003) and the Division of Medical Oncology, Maastricht University Medical Centre, The Netherlands

\section{CONFLICT OF INTEREST}

The authors declare no conflict of interest.

\section{REFERENCES}

Ali D, Le Scodan R (2011) Treatment of the primary tumor in breast cancer patients with synchronous metastases. Ann Oncol 22: 9-16.

Andre F, Slimane K, Bachelot T, Dunant A, Namer M, Barrelier A, Kabbaj O, Spano JP, Marsiglia H, Rouzier R, Delaloge S, Spielmann M (2004) Breast cancer with synchronous metastases: trends in survival during a 14-year period. J Clin Oncol 22: 3302-3308.

Badwe R, Parmar V, Hawaldar R, Nair N, Kaushik R, Siddique S, Navale A, Budrukkar A, Mittra I, Gupta S (2013) Abstract S2-02: Surgical removal of primary tumor and axillary lymph nodes in women with metastatic breast cancer at first presentation: A randomized controlled trial. Cancer Res 73: S2-02-S2-02.

Cardoso F, Costa A, Norton L, Cameron D, Cufer T, Fallowfield L, Francis P, Gligorov J, Kyriakides S, Lin N, Pagani O, Senkus E, Thomssen C, Aapro M, Bergh J, Di Leo A, El Saghir N, Ganz PA, Gelmon K, Goldhirsch A, Harbeck N, Houssami N, Hudis C, Kaufman B, Leadbeater M, Mayer M, Rodger A, Rugo H, Sacchini V, Sledge G, van't Veer L, Viale G, Krop I, Winer E (2012) 1st International consensus guidelines for advanced breast cancer (ABC 1). Breast 21: 242-252.

Chiang AC, Massague J (2008) Molecular basis of metastasis. N Engl J Med 359: 2814-2823.

Dawood S, Broglio K, Buzdar AU, Hortobagyi GN, Giordano SH (2010a) Prognosis of women with metastatic breast cancer by HER2 status and trastuzumab treatment: an institutional-based review. J Clin Oncol 28: 92-98.

Dawood S, Broglio K, Ensor J, Hortobagyi GN, Giordano SH (2010b) Survival differences among women with de novo stage IV and relapsed breast cancer. Ann Oncol 21: 2169-2174.

Dillekas H, Transeth M, Pilskog M, Assmus J, Straume O (2014) Differences in metastatic patterns in relation to time between primary surgery and first relapse from breast cancer suggest synchronized growth of dormant micrometastases. Breast Cancer Res Treat 146: 627-636.

Elston CW, Ellis IO (1991) Pathological prognostic factors in breast cancer. I. The value of histological grade in breast cancer: experience from a large study with long-term follow-up. Histopathology 19: 403-410.

Greene FL, Page DL, Fleming ID, Fritz A, Balch CM, Haller DG, Morrow M (2002) AJCC Cancer Staging Manual. Springer: New York.

Harris JR, Morrow M, Bonadonna G (1993) In Cancer: Principles and Practice of Oncology, pp 1264-1332. Lippincott: Philadelphia.

Harris E, Barry M, Kell MR (2013) Meta-analysis to determine if surgical resection of the primary tumour in the setting of stage IV breast cancer impacts on survival. Ann Surg Oncol 20: 2828-2834.

Kennecke H, Yerushalmi R, Woods R, Cheang MC, Voduc D, Speers CH, Nielsen TO, Gelmon K (2010) Metastatic behavior of breast cancer subtypes. J Clin Oncol 28: 3271-3277.

Largillier R, Ferrero JM, Doyen J, Barriere J, Namer M, Mari V, Courdi A, Hannoun-Levi JM, Ettore F, Birtwisle-Peyrottes I, Balu-Maestro C, Marcy PY, Raoust I, Lallement M, Chamorey E (2008) Prognostic factors in 1,038 women with metastatic breast cancer. Ann Oncol 19: 2012-2019.

Llombart-Cussac A, Pivot X, Biganzoli L, Cortes-Funes H, Pritchard KI, Pierga JY, Smith I, Thomssen C, Srock S, Sampayo M, Cortes J (2014) A prognostic factor index for overall survival in patients receiving first-line chemotherapy for HER2-negative advanced breast cancer: An analysis of the ATHENA trial. Breast 23: 656-662.

Lobbezoo DJ, van Kampen RJ, Voogd AC, Dercksen MW, van den Berkmortel F, Smilde TJ, van de Wouw AJ, Peters FP, van Riel JM, Peters NA, de Boer M, Borm GF, Tjan-Heijnen VC (2013) Prognosis of metastatic breast cancer subtypes: the hormone receptor/HER2-positive subtype is associated with the most favorable outcome. Breast Cancer Res Treat 141: 507-514.

Pal SK, Dehaven M, Nelson RA, Onami S, Hsu J, Waliany S, Kruper L, Mortimer J (2012) Impact of modern chemotherapy on the survival of women presenting with de novo metastatic breast cancer. BMC Cancer 12: 435 .

Puente J, Lopez-Tarruella S, Ruiz A, Lluch A, Pastor M, Alba E, de la Haba J, Ramos M, Cirera L, Anton A, Llombart A, Plazaola A, Fernandez-Aramburo A, Sastre J, Diaz-Rubio E, Martin M (2010) Practical prognostic index for patients with metastatic recurrent breast cancer: retrospective analysis of 2,322 patients from the GEICAM Spanish El Alamo Register. Breast Cancer Res Treat 122: 591-600.

Regierer AC, Wolters R, Ufen MP, Weigel A, Novopashenny I, Kohne CH, Samonigg H, Eucker J, Possinger K, Wischnewsky MB (2014) An internally and externally validated prognostic score for metastatic breast cancer: analysis of 2269 patients. Ann Oncol 25: 633-638.

Rossi V, Nole F, Redana S, Adamoli L, Martinello R, Aurilio G, Verri E, Sapino A, Viale G, Aglietta M, Montemurro F (2014) Clinical outcome in women with HER2-positive de novo or recurring stage IV breast cancer receiving trastuzumab-based therapy. Breast 23: 44-49.

Ruiterkamp J, Voogd AC, Bosscha K, Tjan-Heijnen VC, Ernst MF (2010) Impact of breast surgery on survival in patients with distant metastases at initial presentation: a systematic review of the literature. Breast Cancer Res Treat 120: 9-16.

Slamon DJ, Clark GM, Wong SG, Levin WJ, Ullrich A, McGuire WL (1987) Human breast cancer: correlation of relapse and survival with amplification of the HER-2/neu oncogene. Science 235: 177-182.

Soran A, Ozmen V, Ozbas S, Karanlik H, Muslumanoglu M, Igci A, Canturk Z, Utkan Z, Ozaslan C, Evrensel T (2013) Abstract S2-03: Early follow up of a randomized trial evaluating resection of the primary breast tumor in women presenting with de novo stage IV breast cancer; Turkish study (protocol MF07-01). Cancer Res 73: S2-03-S2-03. 
Weigelt B, Peterse JL, van 't Veer LJ (2005) Breast cancer metastasis: markers and models. Nat Rev Cancer 5: 591-602.

Yamamoto N, Watanabe T, Katsumata N, Omuro Y, Ando M, Fukuda H, Takue Y, Narabayashi M, Adachi I, Takashima S (1998) Construction and validation of a practical prognostic index for patients with metastatic breast cancer. J Clin Oncol 16: 2401-2408.

Yardley DA, Kaufman PA, Brufsky A, Yood MU, Rugo H, Mayer M, Quah C, Yoo B, Tripathy D (2014) Treatment patterns and clinical outcomes for patients with de novo versus recurrent HER2-positive metastatic breast cancer. Breast Cancer Res Treat 145: 725-734.

This work is published under the standard license to publish agreement. After 12 months the work will become freely available and the license terms will switch to a Creative Commons AttributionNonCommercial-Share Alike 4.0 Unported License. 